\title{
The characterization of troponin I levels post synchronised direct-current cardioversion in patients with atrial arrhythmias.
}

\author{
Ryan Sless ${ }^{1,2}$, Gerry Allen ${ }^{3}$, Nathaniel Hayward ${ }^{1,2}$, and Gerry Fahy ${ }^{3}$ \\ ${ }^{1}$ PRN Lab \\ ${ }^{2}$ University College Cork College of Medicine and Health \\ ${ }^{3}$ Cork University Hospital Group
}

April 28, 2020

\begin{abstract}
Cardiac-specific markers of myocardial injury, such as troponin I, are often elevated following procedures that stimulate the myocardium. The aim of this study was to determine the effect of synchronised DC cardioversion of an atrial arrhythmia on myocardial injury 6-hours post procedure, as measured by cardiac troponin I A total of 73 individuals (59 men, 14 women) undergoing DC cardioversion of an atrial arrhythmia agreed to participate in this study. Inclusion criteria included subjects older 18 who were undergoing DC cardioversion for an atrial arrhythmia. This included elective and non-elective admissions. Exclusion criteria included an MI or CABG within the past month, cardioversion for a ventricular arrhythmia, or any recent shocks by an implantable internal cardioverter defibrillator. Patients underwent standard procedure for DC cardioversion with blood work (troponin I and CRP) performed prior to and six hours post cardioversion. Primary outcome was change in troponin I. Secondary outcomes included changes in CRP, relationships between troponin I and cumulative energy and LVM, and a sub-group analysis stratified by the presence of cardiomyopathy. There was no significant change in troponin I following cardioversion $(\mathrm{F}[1,72]=2.651, \mathrm{p}=0.108)$. There was a significant reduction in troponin $\mathrm{I}$ following cardioversion in the non-cardiomyopathy group $(\mathrm{F}[1,58]=6.481, \mathrm{p}=0.014)$. There was no significant relationship between change in troponin $\mathrm{I}$ and cumulative energy or LVM $(\mathrm{r}=0.137, \mathrm{p}=0.306$ and $\mathrm{r}=0.125, \mathrm{p}=0.412$ respectively). Synchronised DC cardioversion of an atrial arrhythmia did not cause myocardial injury 6-hours post-cardioversion. Sub-group analysis suggests that cardioversion of patients with cardiomyopathy may result in normalization of tropo
\end{abstract}

The characterization of troponin I levels post synchronised direct-current cardioversion in patients with atrial arrhythmias.

Ryan Taylor Sless MB BCh BAO,${ }^{1}$ Nathaniel E. Hayward MB BCh BAO,${ }^{1}$ Gerry Allen ANP,${ }^{2}$ Gerry Fahy $\mathrm{MB} \mathrm{BCh}{ }^{2}$

${ }^{1}$ School of Medicine, University College Cork, Cork, Ireland

${ }^{2}$ Department of Cardiology, Cork University Hospital, Cork, Ireland

Corresponding Author:

Dr. Ryan T. Sless

Department of Medicine

University of Toronto

2075 Bayview Avenue

Toronto, Ontario, Canada 
$+1(416) 779-8979$

sless.ryan@gmail.com

Word Count: 2577

\section{Abstract}

\section{Background}

Cardiac-specific markers of myocardial injury, such as troponin I, are often elevated following procedures that stimulate the myocardium. The aim of this study was to determine the effect of synchronised DC cardioversion of an atrial arrhythmia on myocardial injury 6-hours post procedure, as measured by cardiac troponin I

\section{Methods}

A total of 73 individuals (59 men, 14 women) undergoing DC cardioversion of an atrial arrhythmia agreed to participate in this study. Inclusion criteria included subjects older 18 who were undergoing DC cardioversion for an atrial arrhythmia. This included elective and non-elective admissions. Exclusion criteria included an MI or CABG within the past month, cardioversion for a ventricular arrhythmia, or any recent shocks by an implantable internal cardioverter defibrillator. Patients underwent standard procedure for DC cardioversion with blood work (troponin I and CRP) performed prior to and six hours post cardioversion. Primary outcome was change in troponin I. Secondary outcomes included changes in CRP, relationships between troponin I and cumulative energy and LVM, and a sub-group analysis stratified by the presence of cardiomyopathy.

\section{Results}

There was no significant change in troponin I following cardioversion $(\mathrm{F}[1,72]=2.651, p=0.108)$. There was a significant reduction in troponin I following cardioversion in the non-cardiomyopathy group $(\mathrm{F}[1,58]=6.481$, $p=0.014)$. There was no significant relationship between change in troponin I and cumulative energy or $\operatorname{LVM}(\mathrm{r}=0.137, \mathrm{p}=0.306$ and $\mathrm{r}=0.125, p=0.412$ respectively $)$.

\section{Conclusion}

Synchronised DC cardioversion of an atrial arrhythmia did not cause myocardial injury 6-hours postcardioversion. Sub-group analysis suggests that cardioversion of patients with cardiomyopathy may result in normalization of troponin I.

Word Count: 250

Keywords: Atrial Arrhythmias, Electrophysiology, Troponin, Cardioversion

\section{Introduction}

It is widely accepted that the cardiac specific enzymes troponin $\mathrm{T}$ and $\mathrm{I}$ are highly specific markers of myocardial injury. These enzymes have been shown to be elevated during acute coronary syndromes such as myocardial infarction, acute heart failure, pulmonary embolism, infectious conditions of the heart including myocarditis and pericarditis, and sepsis (1-4). There is also evidence to suggest that atrial arrhythmias can cause myocardial injury as indicated by elevations in both C-reactive protein (CRP) and cardiac troponin $\mathrm{T}(5,6)$.

Of the various types of atrial arrhythmias, atrial fibrillation is the most common and recent evidence suggests an increasing prevalence and incidence of atrial fibrillation worldwide (7,8). Advances in electrophysiological procedures have resulted in external synchronised electrical direct current (DC) cardioversion becoming the gold-standard treatment for atrial arrhythmias (9). While other procedures that stimulate the myocardium, such as percutaneous coronary intervention, coronary artery bypass grafting, and pulmonary vein embolization have been shown to create an inflammatory response, as represented by an increase in circulatory 
inflammatory and serum cardiac markers $(10,11)$; there is limited data on the acute incidence of myocardial injury following the external DC cardioversion.

Previous studies in animals have suggested that electrical cardioversion can cause myocardial injury (12). However, these studies did not look directly at biochemical markers of myocardial injury. Additionally, it is unknown whether the energy levels used for cardioversion in animal models correspond to those used in humans. In humans, several clinical studies have attempted to characterize the changes in cardiac troponin I following cardioversion however the results are conflicting (13-16). Additionally, some of the data from these studies were collected following the use of a monophasic defibrillator, which has also been shown to cause greater myocardial injury when compared to the current gold-standard biphasic defibrillators $(13,17)$. A recent study investigating the effects of DC cardioversion on myocardial injury as measured by cardiac troponin T showed no significant changes following cardioversion (18); however these results have not been repeated using troponin I.

Therefore, the objectives of this study are to determine if synchronised DC cardioversion of atrial arrhythmias causes myocardial injury six hours post-cardioversion, by measuring the highly sensitive cardiac biomarker cardiac troponin I. Additionally, we aim to determine if there is a relationship between the change in cardiac troponin I and cumulative energy given during cardioversion or left ventricular mass (as measured by echocardiography).

\section{Methods}

\section{Study Design}

This study was a prospective, observational study that assessed biochemical and structural parameters of cardiac function in patients undergoing synchronised DC cardioversion for atrial arrhythmias. Participants underwent a procedure during a single visit to the Department of Cardiology at Cork University Hospital in Cork, Ireland. During this visit blood sampling was obtained prior to and six hours following the patients DC cardioversion.

\section{Participants}

Individuals undergoing synchronised DC cardioversion for atrial arrhythmias at Cork University Hospital in Cork, Ireland were approached for participation in this study. A total of 73 individuals (59 men, 14 women) agreed to participate in the study. Inclusion criteria included all subjects greater than 18 years of age who were undergoing synchronised DC cardioversion for an atrial arrhythmia. This included both elective and non-elective admissions and included patients with atrial fibrillation, atrial flutter, and focal atrial tachycardia. Exclusion criteria included a myocardial infarction within the past month, coronary artery bypass grafting within the past month, cardioversion for a ventricular arrhythmia, and any recent shocks by an implantable internal cardioverter defibrillator. All procedures conformed to the Declaration of Helsinki and were approved by the Clinical Research Ethics Committee at University College Cork. Written and informed consent was obtained from all participants.

Biochemistry and Cardiac Markers

Baseline blood tests, which included a basic electrolyte panel, C-reactive protein, and cardiac troponin I were recorded in all participants. Blood samples were collected by a registered nurse or advanced nurse practitioner into lithium heparin blood bottles. Blood samples were transported to the Cork University Hospital biochemistry laboratory within 15 minutes of being drawn and analysis of blood samples were completed immediately upon receipt. Six hours following DC cardioversion repeat blood samples for CReactive Protein and Cardiac Troponin I quantification were collected via direct venous puncture. The measurement of pre-cardioversion and six hours post was determined by the Third Universal Definition of Myocardial Infarction guidelines on 'Biomarker Detection of Myocardial Injury with Necrosis.'(19)

Synchronised DC Cardioversion 
All patients underwent synchronised DC cardioversion of their arrhythmia's according to standard protocol. In brief, pre-procedural electrocardiogram was obtained for confirmation of the presenting arrhythmia and participants received general anesthesia. A Phillips HeartStart biphasic Defibrillator XL (Phillips, Amsterdam, Netherlands) was used for the procedure with pad placed in the anterior-posterior position. Adhesive gel pads were used for all procedures. Once sedated, a shock of $150 \mathrm{~J}$ was delivered to the patient. If this was sufficient energy to cardiovert to sinus rhythm the procedure was complete. If the patient remained in arrhythmia, a subsequent shock of 200J was delivered up to two more times. Following the third overall attempt, pads were changed and a fourth and final attempt was made with an energy of 200J. The exact energy of the shock delivered and measured impedance was recorded from the rhythm strip of the defibrillator. A successful cardioversion was defined as at least three consecutive beats of sinus rhythm on the rhythm strip following cardioversion.

\section{Echocardiography}

Two-dimensional transthoracic echocardiography was performed in the left lateral decubitus position by a trained echocardiographer (GE Vivid E9 Imaging System, GE Medical; Horton, Norway) using a M4S Matrix Sector Array Probe $(2-5 \mathrm{MHz})$. Standard views (parasternal long-axis and apical two, four and five chamber) were collected and analyzed according to the recommendations of the American Society for Echocardiography $(20,21)$ using a dedicated software program (GE EchoPAC v201, GE, Horton, Norway) LV mass was subsequently calculated using the Devereux formula: LV Mass $=0.8[1.04[([$ LVEDD + IVSd + $\left.\left.\left.\mathrm{PWd}]^{3}-\mathrm{LVEDD}^{3}\right)\right]\right\}+0.6(22)$.

\section{Statistics}

Preliminary analysis of the data showed potential response differences in patients with cardiomyopathy and as such data were stratified accordingly for additional analysis. Statistical analyses were performed using Statistical Package for Social Sciences software v25 (IBM Corporation, Armonk, New York, USA) and GraphPad Prism (GraphPad Software Inc., La Jolla, California, USA). Data was assessed using repeated measures ANOVA and Pearson's correlation coefficients as a full data set and stratified by cardiomyopathy status. Data are reported as mean \pm standard deviation.

\section{Results}

Of the 103 patients approached to participate in the study, 73 patients were recruited ( 25 did not wish to participate, 6 did not meet exclusion criteria), 14 of which were known to have a diagnosed cardiomyopathy. Baseline clinical parameters are shown in Table 1. Of the participants with cardiomyopathy, two had hypertrophic, three dilated, four ischaemic, and five tachycardia induced.

Table 1. Baseline characteristics of whole-group study participants and stratified by the presence of cardiomyopathy.

\begin{tabular}{lll}
\hline & Total $(\mathrm{n}=73)$ & Carc \\
\hline Age & $67 \pm 9$ & $61 \pm$ \\
Weight (kg) & $92.5 \pm 22.7$ & 93.9 \\
Sex (M:F) & $59: 14$ & $13: 1$ \\
Coronary Artery Disease n(\%) & $7(50$ \\
Hypertension n(\%) & $20(27)$ & $4(29$ \\
Diabetes n(\%) & $36(49)$ & $3(21$ \\
Ejection Fraction (\%) & $9(12)$ & $32 \pm$ \\
Presenting Rhythm n(\%) Atrial fibrillation Atrial flutter Focal atrial tachycardia & $49 \pm 11$ & $13(9$ \\
Antiarrhythmic Medication n(\%) Flecainide Propafenone Sotalol Amiodarone None & $32(43) 1(5) 2(3) 5(7) 17(23) 17(23)$ & 002 \\
Cumulative Energy (J) & $228 \pm 148$ & 222 \\
LV Mass (g) & $227.7 \pm 73.6$ & 272. \\
\end{tabular}


Of the 73 patients that underwent synchronised DC cardioversion for an atrial arrhythmia, $100 \%$ achieved sinus rhythm. Fifty-five (75\%) achieved sinus rhythm with a single shock and 7 (9.5\%) cases received three or more shocks. The cumulative energy delivered is displayed in Table 1.

Table 2. Biochemical and cardiac markers before and after cardioversion in patients with and without cardiomyopathy.

\begin{tabular}{lllllll}
\hline & Troponin-Pre & Troponin-Post & p-value & CRP-Pre & CRP-Post & p-value \\
\hline Total $(\mathrm{n}=73)$ & $20.4 \pm 7.9$ & $17.5 \pm 6.5$ & 0.108 & $5.0 \pm 0.83$ & $4.7 \pm 0.77$ & $\mathbf{0 . 0 4}$ \\
Cardiomyopathy $(\mathrm{n}=14)$ & $74.5 \pm 136.6$ & $54.5 \pm 27.9$ & 0.075 & $2.8 \pm 2.4$ & $2.6 \pm 2.4$ & $\mathbf{0 . 0 1 7}$ \\
Non-cardiomyopathy $(\mathrm{n}=59)$ & $6.7 \pm 3.7$ & $6.2 \pm 3.2$ & $\mathbf{0 . 0 1 4}$ & $5.4 \pm 7.6$ & $5.2 \pm 7.0$ & 0.078 \\
\hline
\end{tabular}

All laboratory samples taken were analyzed by the biochemistry laboratory, with no reports of any samples being haemolysed. The upper reference limit of cardiac troponin I was $>34 \mathrm{ng} / \mathrm{L}$ with the minimum reporting value of $5 \mathrm{ng} / \mathrm{L}$. The cardiac troponin I and C-reactive protein before and after cardioversion in both cohorts are shown in Table 2 with the differences displayed in Figure 1. Repeated measures ANOVA showed a significant decrease in cardiac troponin I following cardioversion in patients without cardiomyopathy $(\mathrm{F}[1,58]=6.481, p=0.014)$. There was also a significant reduction in c-reactive protein in all participants $(\mathrm{F}[1,72]=4.313, p=0.04)$ and the cardiomyopathy sub-analysis $(\mathrm{F}[1,13]=7.602, p=0.017)$ following cardioversion (Figure 1).

\section{Hosted file}

image1.emf available at https://authorea.com/users/312284/articles/444505-the-characterizationof-troponin-i-levels-post-synchronised-direct-current-cardioversion-in-patients-with-atrialarrhythmias

Figure 1. Differences in Troponin I (A) and C-Reactive protein (B) before and after cardioversion when stratified by presence of cardiomyopathy.

\section{Hosted file}

image2. emf available at https : //authorea.com/users/312284/articles/444505-the-characterizationof-troponin-i-levels-post-synchronised-direct-current-cardioversion-in-patients-with-atrialarrhythmias

Figure 2. Changes in troponin I from pre- to post-cardioversion as plotted against cumulative energy in Joules (A) and Left Ventricular Mass (B).

When the change in cardiac troponin I from pre- to post-cardioversion is plotted against cumulative energy delivered during cardioversion (Figure 2A) and LV Mass (Figure 2B) there was no significant relationship amongst the small changes observed $(\mathrm{r}=0.137, p=0.306$ and $\mathrm{r}=0.125, p=0.412$ respectively). However, in the cardiomyopathy group (Figure 2 - grey) there are a few select cases that showed significant reductions in cardiac troponin I.

\section{Discussion}

The results of this study indicated that external DC cardioversion did not result in myocardial injury, as measured by cardiac troponin I, even at higher total cumulative energy. When stratifying by patients with cardiomyopathy, there was a statistically significant reduction in cardiac troponin I following cardioversion in the non-cardiomyopathy subgroup; however this was likely not clinically relevant change, as both before and after values were below the threshold of clinical significance. Additionally, when analyzing changes in CRP there was a statistically significant reduction in the whole group analysis and in the cardiomyopathy group following cardioversion. 
When evaluating the changes in cardiac troponin I, we need to consider the clinical relevance of the changes following cardioversion in addition to the statistical significance of the changes. The assay used in this study to detect troponin I had an upper limit of normal of $34 \mathrm{ng} / \mathrm{L}$. As such, any measured value below this is not clinically significant. Although the non-cardiomyopathy group showed a statistically significant reduction in troponin I (Table 2) the difference of less than $1 \mathrm{ng} / \mathrm{L}$ is of no clinical significance. Furthermore, the range of troponin I (5ng/L-26ng/L) revealed that none of the patients had clinically significant elevations in troponin. Alternatively, in the cardiomyopathy group the reduction in troponin I following cardioversion had greater clinical significance. The average troponin pre-cardioversion was above the upper limit of normal, suggesting possible myocardial injury at baseline. This is largely in agreement with the literature, which states that individuals with cardiomyopathy often have elevated troponin at baseline (23, 24). Additionally, given that troponin peaks approximately 12-hours following myocardial injury, the reduction provides evidence that delivery of the electrical current does not promote myocardial injury. Even though these values are not statistically significant, the clinical relevance is hypothesis generating for the role of cardioversions reversion to sinus rhythm in protection of the myocardium in patients suffering from atrial arrhythmias. To our knowledge there is no other evidence in the literature to suggest that cardioversion can result in an improvement in myocardial injury in patients with cardiomyopathy and an atrial arrhythmia.

Regarding the changes in CRP, the observed reductions, although statistically significant, do not likely carry clinical significance. Elevations in CRP have been shown to increase with myocardial injury and be a predictor of severity of myocardial injury (25). However, the specific test used in our study to assess CRP suggests that an elevation above $10 \mathrm{mg} / \mathrm{L}$ is suggestive of mild inflammation. The average values of CRP detected in the whole population and cardiomyopathy group respectively (Table 2) are all below this value and therefore the detected differences are likely not of any clinical importance at the population level.

When comparing changes in cardiac troponin I to cumulative energy delivered during cardioversion, and left ventricular mass, no relationships were observed. This suggests that increased energy delivered during cardioversion does not injure the myocardium in a dose-dependent manner. Current guidelines for cardioversion recommend the initial shock to be delivered at 150J, only increasing the energy if the first shock failed to revert to sinus rhythm. This guideline is based on animal studies that create uncertainty over the relationship between higher energies and the potential for myocardial injury (26). Since we have shown that increased cumulative energy does not likely cause myocardial injury, the usage of higher energy shocks should be considered for emergent cardioversions in order to increase the likelihood of successful cardioversion (27).

This study has allowed for several hypotheses to be drawn when considering its clinical relevance. Firstly, it confirms the results from Lobo et. al (2018) who reported no evidence of myocardial injury following DC cardioversion by measuring changes in cardiac troponin $\mathrm{T}$, hold true when assessing cardiac troponin I in an alternate cohort. Additionally, it reaffirms their conclusion that an elevated troponin following cardioversion should not be considered a typical or benign result of the procedure itself but may suggest a more serious myocardial pathology (e.g. myocardial infarction) (18) and thus an increased level of suspicion is required. Additionally, this study is hypothesis generating as it suggests that cardioversion in patients with cardiomyopathy who present to hospital with significant myocardial injury, as measured by severely elevated troponin I, may have a greater overall benefit from the return to sinus rhythm. It is possible that there is an additive effect of the cardiomyopathy and abnormal atrial arrhythmia that contributes towards myocardial injury.

This study has several notable strengths but is also not without limitations. Firstly, we observed clinically significant reductions in cardiac troponin I following cardioversion in the patients with cardiomyopathy however our sample size may be underpowered to detect statistically significant differences. Additionally, our study was only single centre and thus subject to possible population bias and limitations with generalizability of the results. Lastly, the Third Universal Definition guidelines on 'Biomarker Detection of Myocardial Injury' suggest that cardiac troponin peaks approximately 12 hours following an MI (21), which may limit the internal validity of this study as we used a time-point of six hours post-procedure to collect hematologic samples. 
Further studies should focus on recruiting more individuals with cardiomyopathies and atrial arrhythmias to determine if cardioversion can result in an improvement in myocardial injury in as short as six hours post-procedure. Additionally, further time points post-cardioversion, up to 12 hours, should be assessed to determine if peak changes in troponin I occur after the six-hour time point.

\section{Conclusion}

In conclusion, synchronised DC cardioversion of an atrial arrhythmia did not cause myocardial injury 6-hours post-cardioversion, as measured by the highly sensitive cardiac troponin I. Sub-group analysis stratifying by the presences of a cardiomyopathy suggests that cardioversion of patients with a cardiomyopathy may result in normalization of troponin I and thus an improvement in myocardial strain or injury. These data suggest that elevations in troponin post-cardioversion should be taken as a serious complication and investigated further. Finally, it may be of benefit for patients with cardiomyopathy and atrial arrhythmias to have their cardioversion more urgently as it may help to improve myocardial injury.

\section{References}

1. Dhoot GK, Gell PG, Perry SV. The localization of the different forms of troponin I in skeletal and cardiac muscle cells. Exp Cell Res. 1978;117(2):357-70.

2. Dhoot GK, Perry SV. Distribution of polymorphic forms of troponin components and tropomyosin in skeletal muscle. Nature. 1979;278(5706):714-8.

3. Knayzer B, Abramov D, Natalia B, Tovbin D, Ganiel A, Katz A. Atrial fibrillation and plasma troponin I elevation after cardiac surgery: relation to inflammation-associated parameters. Journal of cardiac surgery. 2007;22(2):117-23.

4. Oltrona L, Ottani F, Galvani M, Italian Working Group on Atherosclerosis T, Vascular B, the Associazione Nazionale Medici Cardiologi O. Clinical significance of a single measurement of troponin-I and C-reactive protein at admission in 1773 consecutive patients with acute coronary syndromes. Am Heart J. 2004;148(3):405-15.

5. Iakobishvili Z, Weissler A, Buturlin K, Goldenberg G, Strassberg B, Tur R, et al. High Sensitivity Cardiac Troponin T Levels after Elective Cardioversion for Atrial Fibrillation/Flutter. The Israel Medical Association journal : IMAJ. 2015;17(10):607-11.

6. Chung MK, Martin DO, Sprecher D, Wazni O, Kanderian A, Carnes CA, et al. C-reactive protein elevation in patients with atrial arrhythmias: inflammatory mechanisms and persistence of atrial fibrillation. Circulation. 2001;104(24):2886-91.

7. Lip GYH, Brechin CM, Lane DA. The global burden of atrial fibrillation and stroke: a systematic review of the epidemiology of atrial fibrillation in regions outside North America and Europe. Chest. 2012;142(6):148998.

8. Chugh SS, Havmoeller R, Narayanan K, Singh D, Rienstra M, Benjamin EJ, et al. Worldwide epidemiology of atrial fibrillation: a Global Burden of Disease 2010 Study. Circulation. 2014;129(8):837-47.

9. Hernandez-Madrid A, Svendsen JH, Lip GY, Van Gelder IC, Dobreanu D, Blomstrom-Lundqvist C, et al. Cardioversion for atrial fibrillation in current European practice: results of the European Heart Rhythm Association survey. Europace : European pacing, arrhythmias, and cardiac electrophysiology : journal of the working groups on cardiac pacing, arrhythmias, and cardiac cellular electrophysiology of the European Society of Cardiology. 2013;15(6):915-8.

10. Januzzi JL, Jr. Troponin testing after cardiac surgery. HSR Proc Intensive Care Cardiovasc Anesth. 2009;1(3):22-32.

11. Okmen E, Cam N, Sanli A, Unal S, Tartan Z, Vural M. Cardiac troponin I increase after successful percutaneous coronary angioplasty: predictors and long-term prognostic value. Angiology. 2006;57(2):161-9. 
12. Guensch DP, Yu J, Nadeshalingam G, Fischer K, Shearer J, Friedrich MG. Evidence for Acute Myocardial and Skeletal Muscle Injury after Serial Transthoracic Shocks in Healthy Swine. PLoS One. 2016;11(9):e0162245.

13. Kosior DA, Opolski G, Tadeusiak W, Chwyczko T, Wozakowska-Kaplon B, Stawicki S, et al. Serum troponin I and myoglobin after monophasic versus biphasic transthoracic shocks for cardioversion of persistent atrial fibrillation. Pacing Clin Electrophysiol. 2005;28 Suppl 1:S128-32.

14. Piechota W, Gielerak G, Ryczek R, Kazmierczak A, Bejm J, Piechota W. Cardiac troponin I after external electrical cardioversion for atrial fibrillation as a marker of myocardial injury-a preliminary report. Kardiol Pol. 2007;65(6):664-9; discussion 70-1.

15. Cemin R, Rauhe W, Marini M, Pescoller F, Pitscheider W. Serum troponin I level after external electrical direct current synchronised cardioversion in patients with normal or reduced ejection fraction: no evidence of myocytes injury. Clinical cardiology. 2005;28(10):467-70.

16. Santos ES, Pereira MP, Minuzzo L, Moreira DA, Ramos R, Avezum A, et al. [Electrical cardioversion and myocardial injury: evaluation by new cardiac injury markers]. Arquivos brasileiros de cardiologia. 2006;86(3):191-7.

17. Faddy SC, Powell J, Craig JC. Biphasic and monophasic shocks for transthoracic defibrillation: a meta analysis of randomised controlled trials. Resuscitation. 2003;58(1):9-16.

18. Lobo R, Jaffe AS, Cahill C, Blake O, Abbas S, Meany TB, et al. Significance of High-Sensitivity Troponin T After Elective External Direct Current Cardioversion for Atrial Fibrillation or Atrial Flutter. The American journal of cardiology. 2018;121(2):188-92.

19. Thygesen K, Alpert JS, Jaffe AS, Simoons ML, Chaitman BR, White HD, et al. Third universal definition of myocardial infarction. Journal of the American College of Cardiology. 2012;60(16):1581-98.

20. Lang RM, Bierig M, Devereux RB, Flachskampf FA, Foster E, Pellikka PA, et al. Recommendations for chamber quantification: a report from the American Society of Echocardiography's Guidelines and Standards Committee and the Chamber Quantification Writing Group, developed in conjunction with the European Association of Echocardiography, a branch of the European Society of Cardiology. J Am Soc Echocardiogr. 2005;18(12):1440-63.

21. Voigt JU, Pedrizzetti G, Lysyansky P, Marwick TH, Houle H, Baumann R, et al. Definitions for a common standard for 2D speckle tracking echocardiography: consensus document of the EACVI/ASE/Industry Task Force to standardize deformation imaging. J Am Soc Echocardiogr. 2015;28(2):183-93.

22. Jafary FH. Devereux formula for left ventricular mass-be careful to use the right units of measurement. J Am Soc Echocardiogr. 2007;20(6):783.

23. Sato Y, Taniguchi R, Nagai K, Makiyama T, Okada H, Yamada T, et al. Measurements of cardiac troponin T in patients with hypertrophic cardiomyopathy. Heart (British Cardiac Society). 2003;89(6):65960.

24. Barison A, ;, EPastromerlo LE, Giannoni A. Troponin in non-ischaemic dilated cardiomyopathy. European Cardiology Review. 2011;7(3).

25. Dedobbeleer C, Melot C, Renard M. C-reactive protein increase in acute myocardial infarction. Acta cardiologica. 2004;59(3):291-6.

26. Jacobs I, Sunde K, Deakin CD, Hazinski MF, Kerber RE, Koster RW, et al. International Consensus on Cardiopulmonary Resuscitation and Emergency Cardiovasular Care Science With Treatment Recommendations. . Circulation. 2010.

27. Berg RA, Hemphill R, Abella BS, Aufderheide TP, Cave DM, Hazinski MF, et al. Part 5: adult basic life support: 2010 American Heart Association Guidelines for Cardiopulmonary Resuscitation and Emergency Cardiovascular Care. Circulation. 2010;122(18 Suppl 3):S685-705. 
A

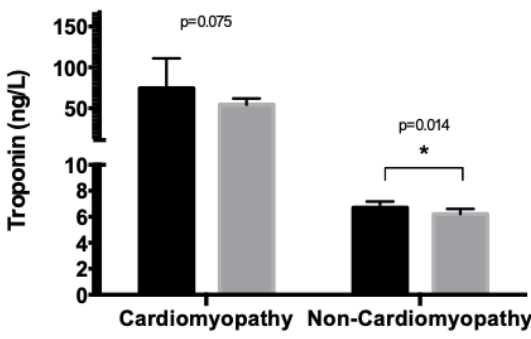

Pre-Cardioversion

A

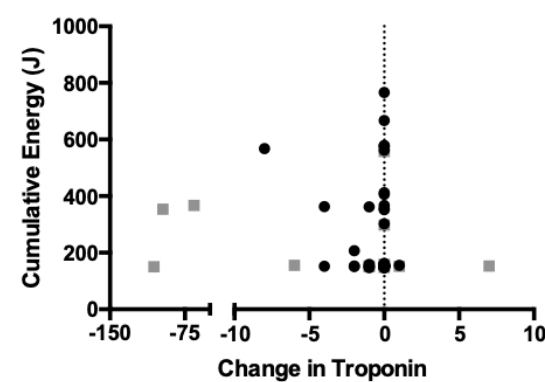

B

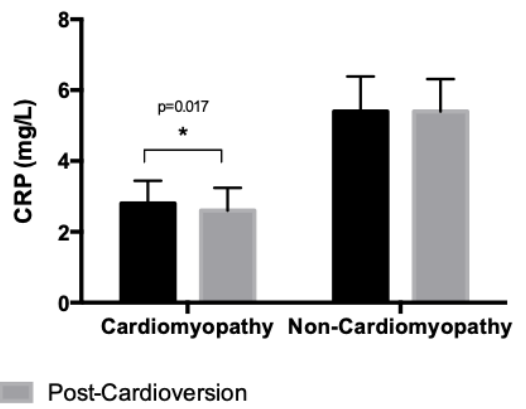

B

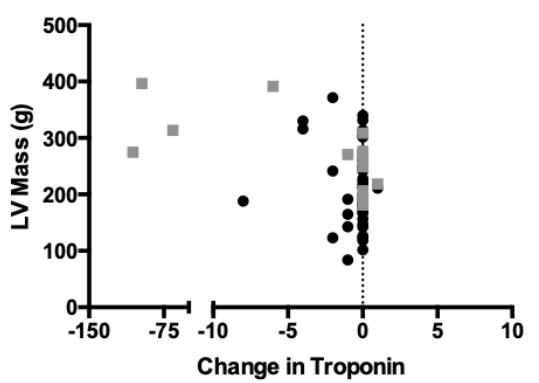

- Cardiomyopathy

- Non-cardiomyopathy 